\title{
In vitro effect of moxifloxacin and rifampicin on biofilm formation by clinical MRSA isolates
}

\author{
Ozturk I ${ }^{1}$, Yurtman $\mathrm{AN}^{1}$, Erac B ${ }^{1}$, Gul-Yurtsever $\mathrm{S}^{2}$, Ermertcan $\mathrm{S}^{1}$, Hosgor-Limoncu $\mathrm{M}^{1}$
}

Ege University, Faculty of Pharmacy, Department of Pharmaceutical Microbiology, Izmir, Turkey. ismail.ozturk@ege.edu.tr

\begin{abstract}
Objective: In this study, it was aimed to investigate in vitro activity of moxifloxacin and rifampicin on biofilm formation by clinical MRSA isolates.

Background: Methicillin resistant Staphylococcus aureus (MRSA) strains could be the causative agent in chronical and medical device associated infections by biofilm formation.

Methods: Moxifloxacin and rifampicin MIC values of 98 MRSA clinical isolates were determined by microdilution method. Biofilm formation of all isolates was determined in 96 -well microplates by using spectrophotometric method. Effects of MIC and sub-inhibitory concentrations (1/2 and 1/4 MIC) of antibiotics on biofilm formation were examined in 46 strong biofilm producer strains.

Results: Biofilm production decreased in 37 and 44 isolates at all studied concentrations of moxifloxacin and rifampicin, respectively. Biofilm production increased in six isolates with moxifloxacin and in two isolates with rifampicin. Coclusion: Biofilm inhibitory effect of rifampicin was found to be stronger than moxifloxacin in the examined strains. The studied antimicrobials also induced biofilm formation in some strains. Results of this study may help to evaluate the effects of these antibiotics on biofilm formation of clinical MRSA strains and to control the antibiotic resistance in clinical settings (Tab. 2, Ref. 25). Text in PDF www.elis.sk.

Key words: biofilm, methicillin resistant Staphylococcus aureus, moxifloxacin, rifampicin.
\end{abstract}

Staphylococci cause a large percentage of infections in damaged tissues, medical implants and catheters by forming biofilms (1). Methicillin-resistant Staphylococcus aureus (MRSA) has become endemic in hospitals worldwide, after first being reported in 1961 (2). MRSA is also a prevalent agent of community acquired infections as well (2-4). MRSA is a multidrug-resistant pathogen and mostly resistant to macrolides, lincosamids, aminoglycosides and beta-lactam agents (5).

Biofilms are defined as an organized community of bacteria, adherent to a surface and contained in an extracellular polymeric substance made of exopolysaccharides, nucleic acids and proteins (6). In the biofilm layer, bacteria are less susceptible to phagocytic macrophages and are resistant to antibiotics, because of their exopolysaccharide matrix and reduced metabolic rate $(6,7)$. The role of biofilm in many diseases is recognized by recent studies. The presence of biofilms has been shown in several chronic diseases including chronic otitis media, cholesteatoma and chronic adenoiditis $(8,9)$.

Fluoroquinolones and rifampicin have been used to treat bacterial infections caused by Gram-positive bacteria including $S$.

${ }^{1}$ Ege University, Faculty of Pharmacy, Department of Pharmaceutical Microbiology, Izmir, Turkey, and ${ }^{2}$ Izmir Katip Celebi University, Department of Medical Microbiology, Ataturk Training and Research Hospital, Izmir, Turkey

Address for correspondence: I. Ozturk, Ege University, Faculty of Pharmacy, Department of Pharmaceutical Microbiology, Izmir, Turkey. Phone: +902323111358, Fax: +902323885258

Acknowledgements: We wish to thank Deniz Ogut and Erkan Azizoglu for their technical supports. aureus. Quinolone and rifampicin resistance is frequently encountered in MRSA strains $(5,10)$.

Although there are many studies which are investigating biofilm formation by different bacteria in the literature, little is known about biofilm formation of clinical MRSA isolates.

In this study, it was aimed to investigate in vitro activity of moxifloxacin and rifampicin on biofilm formation in MRSA strains isolated from clinical samples.

\section{Methods}

\section{Bacterial strains}

Ninety eight MRSA strains were examined in this study. Clinical isolates were recovered from patients at Bacteriology Laboratory, Department of Medical Microbiology, Ataturk Training and Research Hospital, Izmir. Ninety eight MRSA strains had been isolated from wound (33), blood (30), tracheal aspirate (15), sputum (13), urine (2), cerebrospinal fluid (2), nose (2) and throat (1). The strains were stored in brain-heart infusion broth (BHIB) (Merck) with $10 \%$ glycerine at $-80{ }^{\circ} \mathrm{C}$.

\section{Antibiotic susceptibility testing and determination of MIC}

Antibiotic susceptibilities of the studied isolates and minimum inhibitory concentrations (MIC)'s of moxifloxacin (Bayer, Istanbul, Turkey) and rifampicin (Kocak Farma, Istanbul, Turkey) were determined by the disc diffusion method and broth microdilution method, respectively, according to Clinical Laboratory Standards Institute (CLSI)'s recommendations (11). Bacteria were cultured 
on a Mueller-Hinton Agar (MHA) (Merck) at $37^{\circ} \mathrm{C}$ for 24 hours. Bacteria suspensions were adjusted to $0.5 \mathrm{McF}$ arland turbidity and diluted in ratio $1 / 100$. Serial dilutions of antibiotics were distributed to 96 well-microplate. Bacterial suspensions were added to the wells. After incubation at $37^{\circ} \mathrm{C}$ for 24 hours, MIC values were determined. Staphylococcus aureus ATCC 29213 was used as a control strain.

\section{Biofilm formation and quantification}

Bacterial strains were cultured on blood agar (Merck) at $37^{\circ} \mathrm{C}$ for 24 hours. One colony of each bacteria inoculated in $2 \mathrm{ml} \mathrm{BHIB}$ including $2.5 \%$ glucose at $37{ }^{\circ} \mathrm{C}$ for 24 hours. $50 \mu \mathrm{l}$ of bacterial suspensions which were adjusted to $0.5 \mathrm{Mc}$ Farland turbidity, were added into the wells of microplates that contained $150 \mu \mathrm{BHIB}$ and were incubated at $37{ }^{\circ} \mathrm{C}$ for 24 hours. Crystal violet staining method was used as previously described by Bendouah et al (2006) with minor modification, using ethanol (80\%) and acetone (20\%) as bleaching solution. Spectrophotometric measurement was performed at $570 \mathrm{~nm}$. Four dilutions $(1 / 1,1 / 10,1 / 100,1 / 1000)$ of bacterial suspensions were tested to observe the relation between the biofilm production capacity and bacterial concentration. All the procedures were performed in triplicate. Enterococcus faecalis ATCC 29212 was used as a positive control strain. Biofilm formation capacities of the isolates were determined with following criteria:

Optical density (OD) $<1$ negative $(-)$

$$
\begin{aligned}
& 1<\text { OD }<2 \text {; poor positive }(+) \\
& 2<\text { OD }<3 \text {; intermediate positive }(++) \\
& 3<\text { OD; strong positive }(+++)
\end{aligned}
$$

Effect of MIC and sub-MICs of antibiotics on biofilm formation

Biofilm formation capacities of 46 strong biofilm producer strains were determined by the same method in the presence of different concentrations (MIC, 1/2 and 1/4 MIC) of moxifloxacin and rifampicin

\section{Statistical analysis}

Effects of different concentrations of antibiotics on biofilm formation were evaluated by the SPSS-PASW (Statistics 18) program. All data were statistically analysed by T test. $\mathrm{p}$ values smaller than 0.05 were considered to be significant.

\section{Results}

\section{Antibiotic Susceptibility Profiles}

The resistance rates of 98 MRSA strains for trimethoprimsulfamethoxazole, gentamicin, clindamycin, erythromycin, tetracycline, moxifloxacin, rifampicin and ciprofloxacin were 4.2 $\%, 4.3 \%, 53.6 \%, 68 \%, 82.2 \%, 96.9 \%, 96.9 \%$ and $98.9 \%$, respectively. All the strains were susceptible to vancomycin and linezolid.

Ninety five strains were found resistant to moxifloxacin and rifampicin. Three strains were found susceptible to moxifloxacin ( strain no 21, 25 and 97) and rifampicin (strain no 8, 25 and 41). Intermediate strains were regarded as resistant. Moxifloxacin and rifampicin MIC values of 98 strains were shown in Table 1.
Tab. 1. Moxifloxacin and rifampicin MIC values of 98 MRSA strains.

\begin{tabular}{lccc}
\hline $\begin{array}{l}\text { Number } \\
\text { of strains }\end{array}$ & $\begin{array}{c}\text { Moxifloxacin } \\
\text { MIC value }(\mu \mathrm{g} / \mathrm{ml})\end{array}$ & $\begin{array}{c}\text { Number } \\
\text { of strains }\end{array}$ & $\begin{array}{c}\text { Rifampicin } \\
\text { MIC value }(\mu \mathrm{g} / \mathrm{ml})\end{array}$ \\
\hline 2 & 0.064 & 1 & 0.016 \\
1 & 0.256 & 2 & 0.064 \\
1 & 1 & 1 & 2 \\
22 & 2 & 3 & 32 \\
45 & 4 & 3 & 64 \\
19 & 8 & 23 & 128 \\
8 & 16 & 60 & 256 \\
& & 5 & 512 \\
\hline
\end{tabular}

Tab. 2. Effects of moxifloxacin on biofilm formation of nine strains.

\begin{tabular}{lccc}
\hline Strain no & MIC & MIC/2 & MIC/4 \\
\hline 6 & $\uparrow$ & $\downarrow$ & $\downarrow$ \\
15 & $\uparrow$ & $\uparrow$ & $\uparrow$ \\
16 & $\uparrow$ & $\uparrow$ & $\uparrow$ \\
18 & $\uparrow$ & $\uparrow$ & $\uparrow$ \\
19 & $\uparrow$ & $\uparrow$ & $\uparrow$ \\
22 & $\uparrow$ & $\uparrow$ & $\downarrow$ \\
56 & $\uparrow$ & $\downarrow$ & $\downarrow$ \\
57 & $\uparrow$ & $\uparrow$ & $\uparrow$ \\
58 & $\uparrow$ & $\uparrow$ & $\uparrow$ \\
\hline
\end{tabular}

\section{Biofilm formation}

According to the criteria of biofilm production, one MRSA strain (1\%) was classified as negative, 19 MRSA strains (19.3\%) were classified as poor positive, 32 MRSA strains (32.6\%) were classified as intermediate positive and 46 MRSA strains (46.9\%) were classified as strong positive biofilm producers. Among 98 MRSA strains; 23, 29, 36 and 10 strains were produced maximum biofilm for the $1 / 1,1 / 10,1 / 100$ and $1 / 1000$ dilutions, respectively. The strong positive biofilm producing MRSA strains were selected for further studies.

\section{Effect of MIC and sub-MIC of antibiotics on biofilm formation}

Interaction between biofilm formation and different moxifloxacin and rifampicin concentrations (MIC, MIC/2, MIC/4) was investigated with 46 strong biofilm producer isolates. This part of the study was performed with the bacterial dilutions at which maximum biofilm production was observed.

\section{Moxifloxacin}

In 37 of 46 isolates, biofilm production decreased by all studied concentrations of moxifloxacin (in comparison with the control) $(p<0.01)$. Interestingly, by addition of different concentrations of moxifloxacin, biofilm production rates increased in 9 out of 46 isolates (Tab. 2). A variable reduction of biofilm formation by the effect of moxifloxacin at MIC/2 and MIC/4 in two strains (strain no 6 and 56) and at MIC/4 in one strain (strain no 22) was observed when compared to the control. The results indicate that there is no significant difference between MIC-MIC/2 and MIC-MIC/4 of moxifloxacin $(\mathrm{p}>0.05)$.

\section{Rifampicin}

In 44 of 46 isolates, biofilm production was decreased significantly by all studied concentrations of rifampicin in comparison 
with the control $(\mathrm{p}<0.01)$. By addition of MIC/4 of rifampicin, biofilm production rate was increased in two (strain no 25 and $64)$ out of 46 isolates. The results indicate that no significant difference was found between $\mathrm{MIC}$ and $\mathrm{MIC} / 2$, there are some significant differences between MIC-MIC/4 $(\mathrm{p}<0.05)$ and MIC/2$\mathrm{MIC} / 4(\mathrm{p}<0.01)$.

\section{Discussion}

Biofilm, which makes microorganisms more resistant to antibiotics, disinfectants and host immune system, is one of the important virulence factors of microorganisms. Biofilm-forming bacteria have lower sensitivity to antibiotics and phagocytic macrophages due to exopolysaccharide structures and reduced metabolic activity. Many species of bacteria produce biofilm. For example, staphylococci cause serious infections by forming biofilms on damaged tissues, medical implants and vascular catheters $(6-8,12,13)$.

Several different methods such as light and fluorescence microscopy, bioluminescence, Congo red agar and spectrophotometric microplate method could be used for detection of biofilm formation $(7,14,15)$. Spectrophotometric microplate method was found to be more sensitive and specific than other methods and preferred in many studies $(7,15,16)$. In this method, crystal violet stains only bacterial cells not the slimly material. Cells, which are not in the biofilm structure, are rinsed-off by washing steps (14).

Moxifloxacin and rifampicin are used in treatment of infections caused by Gram positive microorganisms. Recent studies reported a tendency of reduction in susceptibilities of staphylococci against moxifloxacin and rifampicin $(17,18)$. In this study, high rates of resistance were found against studied antibiotics except for trimethoprim-sulfamethoxazole and gentamicin. There was no strain resistant to vancomycin and linezolid. In a study of Goldstein et al (17), all MRSA and MSSA strains were found susceptible to vancomycin, rifampin, trimethoprim and sulfamethoxazole. In another study, gentamicin, vancomycin and moxifloxacin resistant staphylococci rates were $26.1 \%, 54.7 \%$ and $76.2 \%$, respectively (18).

Antibiotics have an impact on biofilm production and on mature biofilms. In our study, the results of biofilm formation experiments showed that $79.5 \%$ of MRSA strains were medium or strong positive biofilm producers. It is known that different bacterial dilutions caused different biofilm production rates. Therefore in our study, four different bacterial dilutions were evaluated and it was found that isolates produced the maximum biofilm at 1/100 dilution.

Although microplate-based methods are used in many studies for detection of biofilm formation, implementation of various modifications makes it difficult to compare between the results. Several studies were carried out by modifying the method of Christensen et al $(7,15,16,19)$. In one of these studies, out of 30 MRSA isolates, 9, 11 and 8 isolates were found to be high-level, strong-level and mid-level biofilm producers, respectively (15). Mathur et al (7) investigated 152 clinical staphylococcal isolates with spectrophotometric microplate method described by Christensen et al (19), and found that 22, 60 and 70 of the isolates were strong, medium and poor-negative biofilm producers, respectively. In another study, $30 \%$ of 293 coagulase negative staphylococci
(CNS) isolates were found to be biofilm producer (16). It seems that, biofilm production rates of MRSA isolates are higher than that of MSSA and CNS isolates.

Generally, antibiotics reduce biofilm formation but it was shown that some antibiotics could induce biofilm formation (2022). For example, in our study, the effect of antibiotics on biofilm formation was investigated and studied with the bacterial dilutions at which the maximum biofilm production was observed. Among the examined 46 isolates, biofilm production decreased in 37 and 44 isolates, at all studied concentrations of moxifloxacin and rifampicin, respectively. Nevertheless, biofilm production increased in six and two isolates in the presence of moxifloxacin and rifampicin, respectively. There wasn't any significant difference in the effects of various moxifloxacin concentrations against biofilm formation. Although there wasn't any significant difference between MIC and $\mathrm{MIC} / 2$, there were some significant differences between MIC$\mathrm{MIC} / 4(\mathrm{p}<0.05)$ and MIC/2-MIC/4 $(\mathrm{p}<0.01)$ of rifampicin. Fidan et al (20) examined the effect of different concentrations of ciprofloxacin against biofilm production of $50 \mathrm{CNS}$ isolates. $2 \mathrm{xMIC}$ and MIC values of ciprofloxacin prevented biofilm formation and sub-MIC concentrations significantly decreased biofilm formation.

In accordance with our results, different investigators reported that subminimal inhibitory concentrations of some antibiotics induce biofilm formation. For example, methicillin and cefalexin were shown to stimulate biofilm formation in S. aureus strains $(21,22)$. Also, penicillin, imipenem/tobramycin and cefotaxime were reported to induce biofilm formation in Streptococcus pyogenes, Pseudomonas aeruginosa and Salmonella enterica, respectively (23-25).

To the best of our knowledge, this is the first study reporting the effects of moxifloxacin and rifampicin on biofilm formation in MRSA strains isolated from clinical samples. Biofilm inhibitory activity of rifampicin was found to be stronger than that of moxifloxacin in investigated strains. However, both rifampicin and moxifloxacin also induced biofilm formation in some strains. These results may help to evaluate the effects of antibiotics on biofilm formation of clinical MRSA strains and to control the antibiotic resistance.

\section{References}

1. El-Azizi M, Rao S, Kanchanapoom T, Khardori N. In vitro activity of vancomycin, quinupristin/dalfopristin, and linezolid against intact and disrupted biofilms of staphylococci. Ann Clin Microbiol Antimicrob. 2005; 4: 2. doi:10.1186/1476-0711-4-2.

2. Diep BA, Otto M. The role of virulence determinants in communityassociated MRSA pathogenesis. Trends Microbiol 2008; 16 (8): 361-369.

3. Chambers HF. The changing epidemiology of Staphylococcus aureus? Emerg Infect Dis 2001; 7 (2): 178-182.

4. Okuma K, Iwakawa K, Turnidge JD, Grubb WB, Bell JM, O'Brien FG, Coombs GW, et al. Dissemination of new methicillin-resistant Staphylococcus aureus clones in the community. J Clin Microbiol 2002; 40 (11): 4289-4294.

5. Lentino JR, Narita M, Yu VL. New antimicrobial agents as therapy for resistant gram-positive cocci. Eur J Clin Microbiol Infect Dis 2008; 27 (1): 3-15. 
6. Bendouah Z, Barbeau J, Hamad WA, Desrosiers M. Biofilm formation by Staphylococcus aureus and Pseudomonas aeruginosa is associated with an unfavorable evolution after surgery for chronic sinusitis and nasal polyposis. Otolaryngol Head Neck Surg 2006; 134 (6): 991-996.

7. Mathur T, Singhal S, Khan S, Upadhyay DJ, Fatma T, Rattan A. Detection of biofilm formation among the clinical isolates of Staphylococci: an evaluation of three different screening methods. Indian J Med Microbiol 2006; 24 (1): 25-29.

8. Costerton JW, Stewart PS, Greenberg EP. Bacterial biofilms: a common cause of persistent infections. Science 1999; 284 (5418): 1318-1322.

9. Post JC, Stoodley P, Hall-Stoodley L, Ehrlich GD. The role of biofilms in otolaryngologic infections. Curr Opin Otolaryngol Head Neck Surg 2004; 12 (3): 185-190.

10. Lowy FD. Antimicrobial resistance: the example of Staphylococcus aureus. J Clin Invest 2003; 111 (9): 1265-1273.

11. Clinical and Laboratory Standards Institute. M100-S21, Performance standards for antimicrobial susceptibility testing; twenty first informational supplement. Wayne (PA): CLSI; 2011.

12. Costerton W, Veeh R, Shirtliff M, Pasmore M, Post C, Ehrlich G. The application of biofilm science to the study and control of chronic bacterial infections. J Clin Invest 2003; 112 (10): 1466-1477.

13. Hoiby N, Bjarnsholt T, Givskov M, Molin S, Ciofu O. Antibiotic resistance of bacterial biofilms. Int J Antimicrob Agents 2010; 35 (4): 322-332.

14. Stepanovic S, Vukovic D, Hola V, Di Bonaventura G, Djukic S, Cirkovic I, Ruzicka F. Quantification of biofilm in microtiter plates: overview of testing conditions and practical recommendations for assessment of biofilm production by staphylococci. APMIS 2007; 115 (8): 891-899.

15. Atshan SS, Shamsudin MN, Lung LT, Sekawi Z, Ghaznavi-Rad E, Pei CP. Comparative characterisation of genotypically different clones of MRSA in the production of biofilms. J Biomed Biotechnol 2012. doi: $10.1155 / 2012 / 417247$.

16. de Allori MC, Jure MA, Romero C, de Castillo ME. Antimicrobial resistance and production of biofilms in clinical isolates of coagulase-negative Staphylococcus strains. Biol Pharm Bull 2006; 29 (8): $1592-1596$.
17. Goldstein EJ, Citron DM, Warren YA, Tyrrell KL, Rybak MJ. Virulence characteristics of community-associated Staphylococcus aureus and in vitro activities of moxifloxacin alone and in combination against community-associated and healthcare-associated meticillin-resistant and -susceptible S. aureus. J Med Microbiol 2008; 57 (4): 452-456.

18. Singh S, Katiyar R, Kaistha SD. High oxacillin, vancomycin and fluoroquinolone resistance amongst biofilm forming Staphylococcus aureus isolates from ulcerative keratitis infections. Indian J Med Microbiol 2011; 29 (3): 312-313.

19. Christensen GD, Simpson WA, Younger JJ, Baddour LM, Barrett FF, Melton DM, Beachey EH. Adherence of coagulase-negative staphylococci to plastic tissue culture plates: a quantitative model for the adherence of staphylococci to medical devices. J Clin Microbiol 1985; 22 (6): 996-1006

20. Fidan I, Yuksel S, Cetin Gurelik F. Biofilm production of coagulasenegative Staphylococci and effects of ciprofloxacin on biofilm production. Turk Mikrobiyol Cemiy Derg. 2005; 35 :149-152.

21. Haddadin RN, Saleh S, Al-Adham IS, Buultjens TE, Collier PJ. The effect of subminimal inhibitory concentrations of antibiotics on virulence factors expressed by Staphylococcus aureus biofilms. J Appl Microbiol 2010; 108 (4): 1281-1291.

22. Kaplan JB, Izano EA, Gopal P, Karwacki MT, Kim S, Bose JL, Bayles KW et al. Low levels of beta-lactam antibiotics induce extracellular DNA release and biofilm formation in Staphylococcus aureus. MBio 2012; 3 (4): 198-212.

23. Baldassari L, Recchia S, Creti R, Imperi M, Paratacchia M, Orefici G. Effect of antibiotics at subMIC concentration on biofilm formation by Streptococcus pyogenes. 17th European Congress of Clinical Microbiology and Infectious Diseases (ECCMID) \& 25th International Congress of Chemotherapy (ICC) 2007; P608.

24. Karaman M, Firinci F, Arikan Ayyildiz Z, Bahar IH. Effects of imipenem, tobramycin and curcumin on biofilm formation of Pseudomonas aeruginosa strains. Mikrobiyol Bul 2013; 47 (1): 192-194.

25. Majtan J, Majtanova L, Xu M, Majtan V. In vitro effect of subinhibitory concentrations of antibiotics on biofilm formation by clinical strains of Salmonella enterica serovar Typhimurium isolated in Slovakia. J Appl Microbiol 2008; 104 (5): 1294-301. 